\title{
Glass Microfluidics for Passive Chemoattractant Gradient Generation
}

\author{
G. Wright ${ }^{1}$, L. Costa ${ }^{2}$,A. Terekhov ${ }^{2}$, W. Hofmeister ${ }^{2}$, D. Jowhar1, C. Janetopoulos ${ }^{1,3}$
}

1 Department of Biological Sciences, Vanderbilt University

Nashville, Tennessee 37240

2 Center for Laser Applications, University of Tennessee Space Institute

Tullahoma, Tennessee 37388

3 Department of Cell \& Developmental Biology, Vanderbilt University

Nashville, Tennessee 37240

The ability to elicit a chemoattractant gradient from bulk glass or even a cover slip would provide investigators with a whole new set of experimental platforms. Glass has a number of unique features not found with polydimethylsiloxane (PDMS). With glass, it is possible to etch channels and ports down to a few hundred $\mathrm{nm}$. They are also durable, optically superior, easy to unclog and fill, and are reusable. We are developing glass microfluidic devices that have incorporated microfluidic channels using PDMS or glass (Figure 1). Our devices are useful for confocal and epifluorescent microscopes that are outfitted with environmental chambers or bulky equipment that do not permit the use of a micromanipulator as is typically used to create a passive gradient. In addition, on-chip gradient generators will be useful for high throughput chemotaxis screening assays. Lastly, the two basic designs described here are extremely easy to load and use and would be useful in a laboratory teaching environment.

We have also successfully etched microfluidic channels into microscopy cover slips (180 micron thickness) that allow investigators to perform migration assays (Figure 2). These devices have a small port on the top of the cover slip that elicits a passive chemoattractant gradient. A syringe pump is connected to a manifold fused to the coverslip and provides a small bit of positive pressure for gradient formation. These devices provide several advantages, including the ability to reuse the same gradient generating port for multiple experiments while incorporating the same or different removable PDMS devices. In addition, multiple ports could be integrated into the cover slip so that perfusion could be set up within a PDMS device.

Microfluidic devices with built-in chemical concentration gradient forming structures were fabricated and assembled inside an ISO 1000 class clean room. The microfluidic channels were etched on the surface of UV grade fused silica using the femtosecond laser micromachining system described in reference [1]. The femtosecond laser consists of a diode-pumped frequency doubled Neodymium-doped Yttrium Orthovanadate (Nd:YVO4) laser pump (Verdi), a Ti:Sapphire laser oscillator (Tsunami) and a regenerative amplifier (RegA9000). It operates at a center wavelength of $800 \mathrm{~nm}$, with a repetition rate of up to $250 \mathrm{kHz}$, with a measured pulse width of $160 \mathrm{fs}$ (FWHM), and average power of $1.4 \mathrm{~W}$. The laser beam was focused on the surface of the fused silica substrate using a $\mathrm{H} 32 \mathrm{x} / 0.60$ dry microscope objective. The position of the fused silica substrate under the microscope objective was controlled by an Aerotech ANT95 -50 -XY nMT nanopositioning system. This experimental setup was capable of carefully coordinating the alignment and positioning of the design features through software-controlled stages with nanometer precision. Each microfluidic device was patterned onto a fused silica substrate having two chemoattractant delivery ports and one cell delivery port opened through the thickness of the substrate. After laser micromachining, the 
device was washed in a hot $10 \mathrm{M} \mathrm{KOH}$ solution to remove all machining debris. The device was then rinsed in deionized water and dried in a stream of nitrogen. The microfluidic device side of the fused silica substrate was sealed with a Polydimethylsiloxane (PDMS) coated cover glass using the plasma oxidation bonding method [2]. An acrylic delivery manifold was silicone-bonded to the opposite side of the device. The devices were stored in de-ionized water.

\section{References}

[1] O. H. Y. Zalloum, M. Parrish, A. Terekhov, and W. Hofmeister, "An amplified femtosecond laser system for material micro-/nanostructuring with an integrated Raman microscope", Rev. Sci. Instrum. 81-5, (2010).

[2] D. C. Duffy, J. C. McDonald, O. J. A. Schueller, and G. M. Whitesides, "Rapid prototyping of microfluidic systems in poly(dimethylsiloxane)", Anal. Chem. 70-23, 4974-4984 (1998).

[3] This work was supported by the Tennessee Higher Education Commission through a grant to the Center for Laser Applications, University of Tennessee Space Institute and by NIHGM080370 supporting GW and CJ.

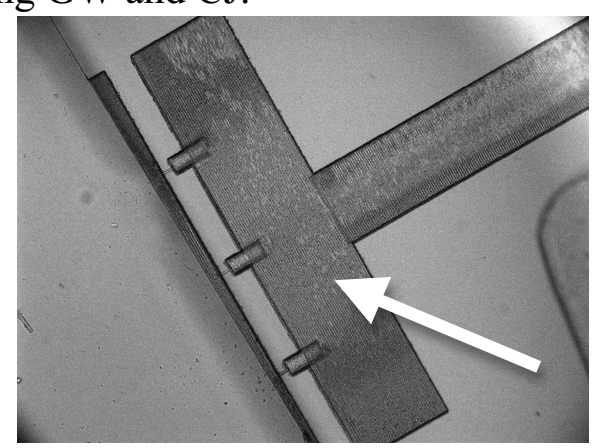

FIG. 1. The bright-field image depicts a glass on-chip microfluidic device containing three laseretched microchannels for gradient generation. The device contains a T-shaped bulk buffer/attractant reservoir channel with three smaller $(\sim 40 \underline{\mu \mathrm{m}})$ reservoir channels attached to three even smaller $(2-5$ $\mu \mathrm{m})$ micropipette outlet channels (white arrow). These micropipette outlet channels connect to the cell loading area (left side of the image).

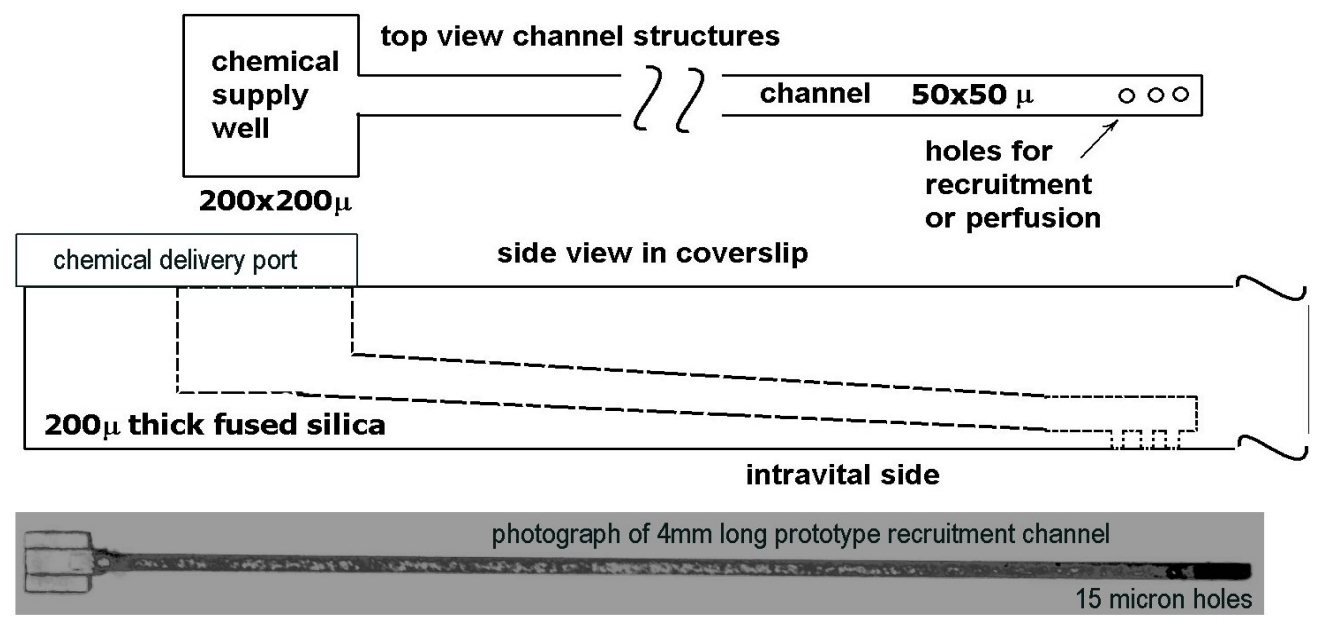

FIG. 2. Gradient generator port and cell recruitment device in a cover slip (objective lens would come from the top). Dimensions and cartoon of microfluidic coverslip (top) and a low magnification image of part of a recently etched device (bottom). It should be noted that the holes are actually in the center of the coverslip, so there is no difficulty bringing in an objective lens. 